\title{
Synthesis and anticancer activity of new carbohydrazide derivatives bearing furan moiety
}

\author{
Fatih TOK $^{1}$ (D), Elif KAYA TİLKİ 2 (D), Miriş DIKMEN ${ }^{2}$ (D) Bedia KOÇYİĞİT-KAYMAKÇIOĞLU ${ }^{1}$ * (D) \\ 1 Department of Pharmaceutical Chemistry, Faculty of Pharmacy, Marmara University, Başıüyük 34854, İstanbul, \\ Turkey. \\ 2 Department of Pharmacology, Faculty of Pharmacy, Anadolu University, Tepebaş1 26210, Eskişehir, Turkey. \\ * Corresponding Author. E-mail: bkaymakcioglu@marmara.edu.tr (B.K.); Tel. +90-216-777 5200.
}

Received: 20 June 2021 / Revised: 26 July 2021 / Accepted: 15 November 2021

\begin{abstract}
In this study, some new carbohydrazide derivatives bearing furan moiety were synthesized. All carbohydrazide structures have been characterized by IR, $1 \mathrm{H}-\mathrm{NMR}$ and elemental analysis. Anticancer activity of compounds were investigated on A549 human lung cancer and BJ normal fibroblast cells. According to the MTT assay results, all compounds demonstrated the cytotoxic activity on A549 cells with $\mathrm{IC}_{50}$ values of 43.38-342.63 $\mu \mathrm{M}$ except compound 3g. Only the $\mathrm{IC}_{50}$ values of compound $3 \mathrm{c}$ was below $400 \mu \mathrm{M}$ on BJ cells. Especially, compound 3e showed significant anticancer effects on A549 cells with $\mathrm{IC}_{50}$ value of $43.38 \mu \mathrm{M}$ and also didn't show cytotoxic effects on normal BJ cells.
\end{abstract}

KEYWORDS: Amide; furan; anticancer; carbohydrazide.

\section{INTRODUCTION}

Cancer is one of the major causes of death worldwide [1]. Lung cancer is one of the most common malignancies, accounting for $11.4 \%$ of all new cancer cases in the world. It was estimated that lung cancer is the second most common cancer after breast cancer and the leading cause of cancer death by the International Agency for Research on Cancer [2]. It has a particularly poor prognosis, leading to more deaths in European Union and the United States of America than any other malignant disease [3]. Development of identification and treatment are important steps for cancer treatment. However, the requirement for effective and selective chemotherapeutic agents continues.

Furan, a five membered heterocyclic ring, consists of four carbon atoms and one oxygen atom [4]. Furan rings are known their impressive optical properties and excellent charge-transport properties [5]. Therefore furan containing compounds display numerous biological and pharmaceutical properties such as antimicrobial, anticancer, antibacterial and analgesic activity [6-8]. 2,5-Disubstituted furan derivatives are found in many natural products and oxygen atom in furan ring can form hydrogen bond with biological enzymes or receptors [9]. The structures of acylhydrazide are seen in many bioactive molecules [10] and they promise a range of pharmaceutical applications because of their two hydrogen bonding and two electron donor group (-CONHNHCO-) [11, 12]. Cui et al., synthesized acylhydrazide derivatives bearing furan ring and reported their significant antitumor activity [13]. Shafeeulla et al., indicated the strong cytotoxic effects of diacylhydrazine structures they synthesized [14]. Besides, Sun et al., chose acylhydrazide derivatives for antitumor activity due to their unique structure and strong enzyme potency [15].

In this study, it was aimed to be able to find effective and selective molecules for lung cancer treatment. Therefore methyl 5-(4-aminophenyl)furan-2-carboxylate was chosen as starting material for this synthesis and new carbohydrazide derivatives were synthesized. Their anticancer activity on A549 human lung cancer and BJ normal fibroblast cells were evaluated by the MTT test.

How to cite this article: Tok F, Kaya Tilki E, Dikmen M, Koçyiğit-Kaymakçığlu B. Synthesis and anticancer activity of new carbohydrazide derivatives bearing furan moiety. J Res Pharm. 2022; 26(1): 13-19. 


\section{RESULTS AND DISCUSSION}

\subsection{Chemistry}

The target compounds were obtained at three different steps. Initially, the substituted benzoyl chloride derivatives and methyl 5-(4-aminophenyl)furan-2-carboxylate were dissolved in tetrahydrofuran. The reaction was completed with stirring on magnetic stirrer for $4 \mathrm{~h}$ at $25^{\circ} \mathrm{C}$. In the second step, the hydrazide molecules were synthesized from the reaction of ester functional group with hydrazine monohydrate in ethanol for $6 \mathrm{~h}$ at $80-90^{\circ} \mathrm{C}$. Finally, the hydrazide compounds were treated with different substituted benzoyl chloride in chloroform to get the target compounds (3a-3g) (Figure 1).<smiles>[Y]C#CC(=O)c1ccc(-c2ccc(NC(=O)c3ccc([R])cc3)cc2)o1</smiles>

Figure 1. Synthetic protocol of novel compounds. Reactant and Reagent: (i) $\mathrm{NaHCO}_{3}$, tetrahydrofuran; (ii) hydrazine monohydrate, ethanol; (iii) chloroform, substituted benzoyl chloride.

The target compounds (3a-3g) were elucidated by IR, ${ }^{1} \mathrm{H}-\mathrm{NMR}$ and elemental analysis. In the IR spectra, the NH stretching bands belonging to amide and hydrazide groups were detected at $3103-3390 \mathrm{~cm}^{-1}$. The strong sharp bands from 1633 to $1697 \mathrm{~cm}^{-1}$ were attributed to $\mathrm{C}=\mathrm{O}$ amide and carbohydrazide stretching vibrations. The aromatic C-H stretching bands were determined at 3003-3041 $\mathrm{cm}^{-1}$. In the ${ }^{1} \mathrm{H}-\mathrm{NMR}$ spectra, three different NH peaks belonging to amide and carbohydrazide groups were observed between $\delta 10.38$ and $10.90 \mathrm{ppm}$. For example, these NH peaks were demonstrated in the ${ }^{1} \mathrm{H}-\mathrm{NMR}$ spectrum of compound $3 \mathbf{e}$ in Figure 2 below. The aromatic protons appeared at $\delta 7.00-8.41 \mathrm{ppm}$ as multiplet peaks. The elemental analysis of target compounds were in accordance with theoretical values.

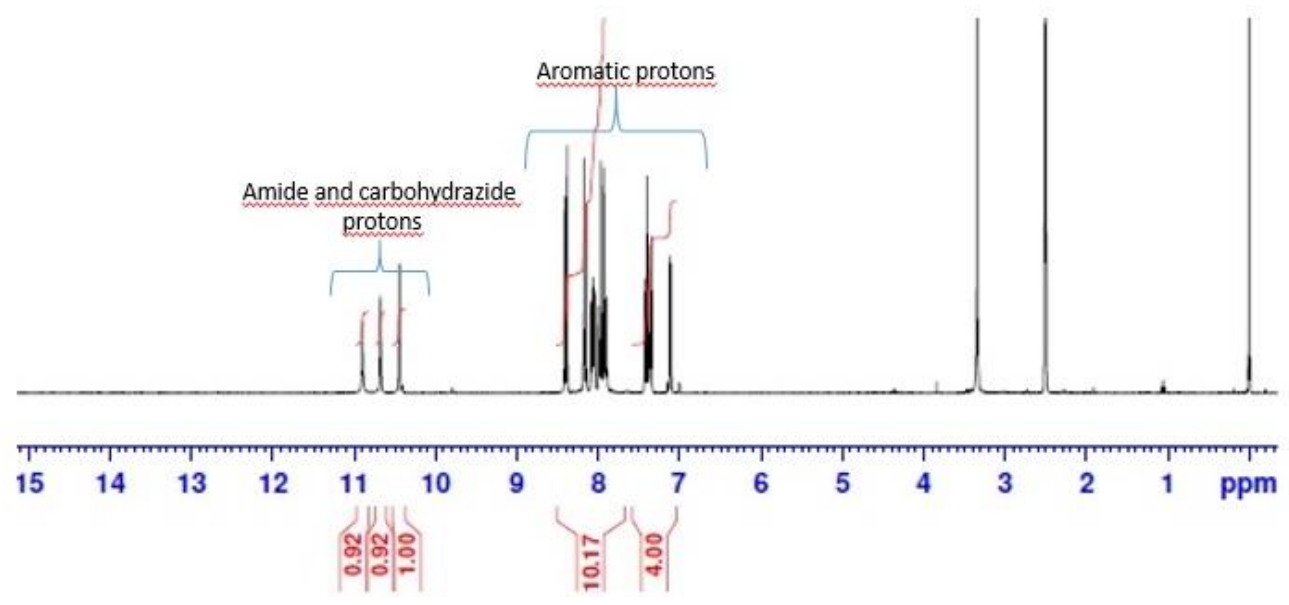

Figure 2 . The ${ }^{1} \mathrm{H}-\mathrm{NMR}$ spectrum of compound $3 \mathbf{e}$. 


\subsection{Biological evaluation}

The anticancer activities of compounds were determined against A549 lung cancer cells and BJ normal fibroblast cells by MTT assay. The $\mathrm{IC}_{50}$ values of compounds on A549 and BJ cells were given in Table 1.

Table 1. $\mathrm{IC}_{50}$ values $(\mu \mathrm{M})$ of the compounds on different cell lines after $24 \mathrm{~h}$ incubation period.

\begin{tabular}{ccccc}
\hline Compound & $\mathbf{R}_{\mathbf{1}}$ & $\mathbf{R}_{\mathbf{2}}$ & \multicolumn{2}{c}{ IC $_{\mathbf{5 0}}$ values } \\
\cline { 4 - 5 } & & & $\mathbf{A 5 4 9}$ & $\mathbf{B J}$ \\
\hline Cyclophosphamide & - & - & 242.41 & $>400$ \\
3a & $\mathrm{F}$ & $\mathrm{OCH}_{3}$ & 115.30 & $>400$ \\
3b & $\mathrm{F}$ & $\mathrm{F}$ & 326.75 & $>400$ \\
3c & $\mathrm{Cl}$ & $\mathrm{CH}_{3}$ & 245.65 & 380.96 \\
3d & $\mathrm{Cl}$ & $\mathrm{OCH}_{3}$ & 176.00 & $>400$ \\
3e & $\mathrm{F}$ & $\mathrm{NO}_{2}$ & 43.38 & $>400$ \\
3f & $\mathrm{F}$ & $\mathrm{Cl}_{3}$ & 342.63 & $>400$ \\
3g & $\mathrm{Cl}$ & $\mathrm{NO}_{2}$ & $>400$ & $>400$ \\
\hline
\end{tabular}

According to the MTT assay results, the A549 lung cancer cell viability was significantly decreased with all compounds but except 3g. None of the compounds showed cytotoxic effects on BJ normal fibroblast cells except compound 3c. Only the $\mathrm{IC}_{50}$ values of compound $3 \mathrm{c}$ was below $400 \mu \mathrm{M}$ on BJ cells. Among these compounds, compound 3e bearing electron withdrawing group $\left(\mathrm{NO}_{2}\right)$ and halogen (fluoro) substituents demonstrated significant anti-cancer effects on human A549 lung cancer cells with $43.38 \mu \mathrm{M} \mathrm{IC}_{50}$ value and also didn't show cytotoxic effects on normal BJ cells. The selectivity index of compounds was also found to be high.

\subsection{ADME properties}

According to Lipinski rules, molecular weight should be lower than 500; the number of hydrogen bond donors and hydrogen bond acceptors should be less than 5 and 10, respectively. The values of theoretical partition coefficient (cLog P) should be less than the maximum value of 5 . Therefore none of the target compounds violated the Lipinski rules. Furthermore, no more than 10 rotatable bonds and topological polar surface area not greater than $140 \AA 2$ should be for ideal drug candidate. On the other hand, water solubility play a critical role in drug's bioavailability. After calculation of Log $S$ values, all compounds were found as moderately soluble (Table 2).

Table 2. Calculated physicochemical properties of the synthesized compounds.

\begin{tabular}{cccccccccc}
\hline Comp. & MW & RB & HA & HD & MR & TPSA & cLog P & Log S & Lipinski \\
\hline 3a & 477.87 & 9 & 5 & 3 & 124.03 & 124.03 & 3.42 & -5.85 & Yes \\
3b & 473.45 & 10 & 6 & 3 & 125.51 & 109.67 & 2.63 & -5.33 & Yes \\
3c & 461.42 & 9 & 4 & 3 & 118.98 & 100.44 & 3.32 & -5.42 & Yes \\
3d & 473.91 & 9 & 4 & 3 & 129.04 & 100.44 & 3.52 & -5.99 & Yes \\
3e & 489.91 & 10 & 5 & 3 & 130.57 & 109.67 & 2.73 & -5.77 & Yes \\
3f & 488.42 & 10 & 7 & 3 & 127.84 & 146.26 & 2.03 & -5.32 & Yes \\
3g & 504.88 & 10 & 6 & 3 & 132.90 & 146.26 & 2.14 & -5.76 & Yes \\
\hline
\end{tabular}

MW: Molecular weight; RB: Number of rotatable bonds; HA: Number of hydrogen acceptors; HD: Number of hydrogen donors; MR: Molar refractivity; TPSA: Topological polar surface area; cLog P: calculated Log P; Log S: Water solubility.

\section{CONCLUSION}

In the present study, we synthesized novel carbohydrazide derivatives bearing furan moiety. All new compounds were characterized by elemental analysis and various spectroscopic methods (FTIR, ${ }^{1} \mathrm{H}-\mathrm{NMR}$ ). Their anticancer activities were investigated on A549 lung cancer cells and normal BJ fibroblasts. The A549 lung cancer cell viability was significantly decreased with all compounds but except compound $3 \mathrm{~g}$ and conversely, these compounds didn't show cytotoxic effects on BJ normal fibroblast cells. Compound 3e containing fluoro and nitro substituent showed the best anticancer activity against A549 cell and no toxicity of normal fibroblast BJ. Therefore, this compound could be a lung cancer drug candidate with further development. 


\section{MATERIALS AND METHODS}

\subsection{Chemistry}

All chemicals and reagents were purchased from Sigma-Aldrich and Merck companies. Thin-layer chromatography (TLC) was performed on Merck silica gel 60 F254 aluminum sheets for the purity of the compounds and the progress of reaction. Melting points were determined using Schmelzpunktbestimmer SMP II basic model. The infrared spectra were recorded on a Shimadzu FTIR 8400 S spectrophotometer. The ${ }^{1} \mathrm{H}-\mathrm{NMR}$ spectra in deutero dimethyl sulfoxide (DMSO- $\left.d_{6}\right)$ using TMS as an internal standard were recorded on a Bruker $300 \mathrm{MHz}$ Ultrashield TM. Elemental analyses of compounds $(\mathrm{C}, \mathrm{H}, \mathrm{N})$ were performed on a VarioMICRO elemental analyzer.

\subsubsection{General procedure of preparation of amide derivatives}

Methyl 5-(4-aminophenyl)furan-2-carboxylate $(1 \mathrm{mmol})$, tetrahydrofuran $(10 \mathrm{~mL})$ and $\mathrm{NaHCO}_{3}(3$ mmol) were taken in a flask. Substituted benzoyl chloride $(1 \mathrm{mmol})$ was added dropwise to solution of $m e t h y l$ 5-(4-aminophenyl)furan-2-carboxylate. The reaction was stirred at room temperature for $4 \mathrm{~h}$. The solvent was evaporated under vacuum. The precipitate was filtered, washed with water and crystallized from methanol [16].

\subsubsection{General procedure of preparation of hydrazide derivatives}

Amide derivative $(1 \mathrm{mmol})$, hydrazine monohydrate $(1 \mathrm{mmol})$ and $15 \mathrm{~mL}$ ethanol was taken in a flask. The reaction was refluxed for 6-8 h. After cooling, the precipitate was filtered and recrystallized from methanol [17].

\section{4-Fluoro-N-(4-(5-(hydrazinecarbonyl)furan-2-yl)phenyl)benzamide}

White solid; Yield: 65\%; m.p. 260-261 ${ }^{\circ} \mathrm{C}$; IR (v, cm²): 3357, 3125 (N-H), 3059 (C-H), 1662 (C=O), 1599, 1573, 1548, 1456 (aromatic C=C, C-N strech. and N-H bend.), 1327 (C-O), 850 (aromatic C-H bend.). ${ }^{1} \mathrm{H}-\mathrm{NMR}$ (300 MHz, DMSO-d $\left.d_{6}\right): \delta 4.48\left(\mathrm{~s}, 2 \mathrm{H}, \mathrm{NH}_{2}\right), 6.99-8.08(\mathrm{~m}, 10 \mathrm{H}, \mathrm{Ar}-\mathrm{H}), 9.79-10.40$ (2s, 2H, NH); Anal. calcd. for $\mathrm{C}_{18} \mathrm{H}_{14} \mathrm{FN}_{3} \mathrm{O}_{3}: \mathrm{C}, 63.71 ; \mathrm{H}, 4.16 ; \mathrm{N}, 12.38$; Found: C, 63.55; H, 4.19; N, $12.44 \%$.

4-Chloro-N-(4-(5-(hydrazinecarbonyl)furan-2-yl)phenyl)benzamide

White solid; Yield: 60\%; m.p. 277-279 ${ }^{\circ} \mathrm{C}$; IR (v, cm $\left.{ }^{-1}\right)$ : 3310, 3109 (N-H), 3030 (C-H), 1660 (C=O), 1595, 1517, 1496, 1456 (aromatic C=C, C-N strech. and N-H bend.), 1315 (C-O), 842 (aromatic C-H bend.). ${ }^{1} \mathrm{H}-\mathrm{NMR}$ (300 MHz, DMSO-d $\left.d_{6}\right): \delta 4.42$ (s, 2H, NH ), 7.03-8.22 (m, 10H, Ar-H), 9.66-10.46 (2s, 2H, NH); Anal. calcd. for $\mathrm{C}_{18} \mathrm{H}_{14} \mathrm{ClN}_{3} \mathrm{O}_{3}$ : C, 60.77; H, 3.97; N, 11.81; Found: C, 60.87; H, 3.94; N, 11.87\%.

\subsubsection{General procedure of preparation of carbohydrazide derivatives}

Substituted benzoyl chloride $(0.5 \mathrm{mmol})$ and hydrazide derivative $(0.5 \mathrm{mmol})$ were taken in a flask. Chloroform $(15 \mathrm{~mL})$ was added into the mixture. The reaction was refluxed for $8 \mathrm{~h}$. The solvent was evaporated under vacuum and the precipitate was filtered and recrystallized from ethanol [18].

4-Fluoro-N-[4-(5-(2-(4-methoxybenzoyl)hydrazinecarbonyl)furan-2-yl)phenyl]benzamide (3a)

Light yellow solid; Yield: 75\%; m.p. 226-227º C; IR (v, cm-1): 3385, 3115 (N-H), 3005 (aromatic C-H), 2975, 2839 (aliphatic C-H), 1687, 1650 (C=O), 1593, 1519, 1487, 1456 (aromatic C=C, C-N strech. and N-H bend.), 1311 (C-O), 835 (aromatic C-H bend.). ${ }^{1} \mathrm{H}-\mathrm{NMR}$ (300 MHz, DMSO-d $)$ : $\delta 3.84$ (s, 3H, OCH $), 7.34-8.02$ (m, $14 \mathrm{H}, \mathrm{Ar}-$ $\mathrm{H}), 10.49-10.63$ (3s, 3H, NH); Anal. calcd. for $\mathrm{C}_{26} \mathrm{H}_{20} \mathrm{FN}_{3} \mathrm{O}_{5}$ : C, 65.96; H, 4.26; N, 8.88; Found: C, 65.80; H, 4.30; N, $8.95 \%$.

4-Fluoro-N-[4-(5-(2-(4-fluorobenzoyl)hydrazinecarbonyl)furan-2-yl)phenyl]benzamide (3b)

Light yellow solid; Yield: 72\%; m.p. 219-220 C; IR (v, cm-1): 3319, 3207 (N-H), 3041 (aromatic C-H), 1687, 1633 (C=O), 1599, 1525, 1496, 1456 (aromatic C=C, C-N strech. and N-H bend.), 1325 (C-O), 831 (aromatic C-H bend.). ${ }^{1} \mathrm{H}-\mathrm{NMR}\left(300 \mathrm{MHz}, \mathrm{DMSO}-d_{6}\right): \delta$ 7.10-8.09 (m, 14H, Ar-H), 10.44-10.57 (3s, 3H, NH); Anal. calcd. for $\mathrm{C}_{25} \mathrm{H}_{17} \mathrm{~F}_{2} \mathrm{~N}_{3} \mathrm{O}_{4}$ : C, 65.08; H, 3.71; N, 9.11; Found: C, 65.25; H, 3.67; N, 9.03\%.

4-Chloro-N-[4-(5-(2-(4-methylbenzoyl)hydrazinecarbonyl)furan-2-yl)phenyl]benzamide (3c) 
Off-white solid; Yield: 69\%; m.p. 234-235다 IR (v, cm-1): 3284, 3111 (N-H), 3006 (aromatic C-H), 2960, 2856 (aliphatic C-H), 1680, 1643 (C=O), 1591, 1531, 1479, 1456 (aromatic C=C, C-N strech. and N-H bend.), 1315 (C-O), 842 (aromatic C-H bend.). ${ }^{1} \mathrm{H}-\mathrm{NMR}\left(300 \mathrm{MHz}, \mathrm{DMSO}-d_{6}\right): \delta 2.30\left(\mathrm{~s}, 3 \mathrm{H}, \mathrm{CH}_{3}\right), 7.00-8.10(\mathrm{~m}, 14 \mathrm{H}, \mathrm{Ar}-\mathrm{H})$, 10.38-10.50 (3s, 3H, NH); Anal. calcd. for $\mathrm{C}_{26} \mathrm{H}_{20} \mathrm{ClN}_{3} \mathrm{O}_{4}$ : C, 65.89; H, 4.25; N, 8.87; Found: C, 65.77; H, 4.23; N, $8.83 \%$.

4-Chloro-N-[4-(5-(2-(4-methoxybenzoyl)hydrazinecarbonyl)furan-2-yl)phenyl]benzamide (3d)

Off-white solid; Yield: 79\%; m.p. 227-228 ${ }^{\circ} \mathrm{C}$; IR (v, cm-1): 3389, 3109 (N-H), 3010 (aromatic C-H), 2950, 2870 (aliphatic C-H), 1697, 1660 (C=O), 1595, 1519, 1459, 1435 (aromatic C=C, C-N strech. and N-H bend.), 1311 (C-O), 835 (aromatic C-H bend.). ${ }^{1} \mathrm{H}-\mathrm{NMR}\left(300 \mathrm{MHz}, \mathrm{DMSO}-d_{6}\right): \delta 3.82\left(\mathrm{~s}, 3 \mathrm{H}, \mathrm{OCH}_{3}\right), 7.11-8.02(\mathrm{~m}, 14 \mathrm{H}, \mathrm{Ar}-$ $\mathrm{H}), 10.50-10.75(3 \mathrm{~s}, 3 \mathrm{H}, \mathrm{NH})$; Anal. calcd. for $\mathrm{C}_{26} \mathrm{H}_{20} \mathrm{ClN}_{3} \mathrm{O}_{5}$ : C, 63.74; $\mathrm{H}, 4.11 ; \mathrm{N}, 8.58$; Found: $\mathrm{C}, 63.79 ; \mathrm{H}, 4.14$; $\mathrm{N}, 8.53 \%$.

\section{4-Fluoro-N-[4-(5-(2-(4-nitrobenzoyl)hydrazinecarbonyl)furan-2-yl)phenyl]benzamide (3e)}

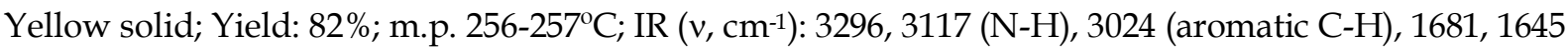
$(\mathrm{C}=\mathrm{O}), 1602,1573,1525,1456$ (aromatic $\mathrm{C}=\mathrm{C}, \mathrm{C}-\mathrm{N}$ strech. and N-H bend.), 1325 (C-O), 825 (aromatic C-H bend.). ${ }^{1} \mathrm{H}-\mathrm{NMR}\left(300 \mathrm{MHz}, \mathrm{DMSO}-d_{6}\right): \delta 7.36-8.41(\mathrm{~m}, 14 \mathrm{H}, \mathrm{Ar}-\mathrm{H}), 10.44-10.89$ (3s, $\left.3 \mathrm{H}, \mathrm{NH}\right)$; Anal. calcd. for $\mathrm{C}_{25} \mathrm{H}_{17} \mathrm{FN}_{4} \mathrm{O}_{6}: \mathrm{C}, 61.48 ; \mathrm{H}, 3.51 ; \mathrm{N}, 11.47$; Found: $\mathrm{C}, 61.62 ; \mathrm{H}, 3.54 ; \mathrm{N}, 11.54 \%$.

4-Fluoro-N-[4-(5-(2-(4-Chlorobenzoyl)hydrazinecarbonyl)furan-2-yl)phenyl]benzamide (3f)

Light yellow solid; Yield: 75\%; m.p. 220-221 ${ }^{\circ} \mathrm{C}$; IR (v, $\left.\mathrm{cm}^{-1}\right)$ : 3390, $3103(\mathrm{~N}-\mathrm{H}), 3010$ (aromatic C-H), 1695, 1658 (C=O), 1595, 1516, 1487, 1456 (aromatic $\mathrm{C}=\mathrm{C}, \mathrm{C}-\mathrm{N}$ strech. and $\mathrm{N}-\mathrm{H}$ bend.), 1323 (C-O), 833 (aromatic C-H bend.). ${ }^{1} \mathrm{H}-\mathrm{NMR}\left(300 \mathrm{MHz}, \mathrm{DMSO}-d_{6}\right): \delta$ 7.09-8.09 (m, $\left.14 \mathrm{H}, \mathrm{Ar}-\mathrm{H}\right), 10.44-10.63$ (3s, 3H, NH); Anal. calcd. for $\mathrm{C}_{25} \mathrm{H}_{17} \mathrm{ClFN}_{3} \mathrm{O}_{4}$ : C, 62.83; H, 3.59; N, 8.79; Found: C, 63.07; H, 3.55; N, 8.85\%.

4-Chloro-N-[4-(5-(2-(4-nitrobenzoyl)hydrazinecarbonyl)furan-2-yl)phenyl]benzamide (3g)

Yellow solid; Yield: 84\%; m.p. 212-213우 ${ }^{\circ}$ IR (v, cm-1): 3389, 3115 (N-H), 3003 (aromatic C-H), 1677, 1660 $(\mathrm{C}=\mathrm{O}), 1595,1573,1516,1456$ (aromatic $\mathrm{C}=\mathrm{C}, \mathrm{C}-\mathrm{N}$ strech. and N-H bend.), 1311 (C-O), 812 (aromatic C-H bend.). ${ }^{1} \mathrm{H}-\mathrm{NMR}\left(300 \mathrm{MHz}, \mathrm{DMSO}-d_{6}\right): \delta 7.42-8.41(\mathrm{~m}, 14 \mathrm{H}, \mathrm{Ar}-\mathrm{H}), 10.49-10.90(3 \mathrm{~s}, 3 \mathrm{H}, \mathrm{NH})$; Anal. calcd. for $\mathrm{C}_{25} \mathrm{H}_{17} \mathrm{ClN}_{4} \mathrm{O}_{6}$ : C, 59.47; H, 3.39; N, 11.10; Found: C, 59.32; H, 3.42; N, 11.14\%.

\subsection{Biological Activity}

\subsubsection{Cell culture}

The human lung cancer A549 (ATCC® CCL-185'T) and human normal fibroblast BJ (ATCC® CRL$2522^{\mathrm{TM}}$ ) cell lines were obtained from ATCC $($ and maintained in $10 \%$ fetal bovine serum and $1 \%$ penicillin/streptomycin containing RPMI (A549) or EMEM (BJ) medium at $37^{\circ} \mathrm{C}$ in a humidified incubator with $5 \% \mathrm{CO}_{2}$.

\subsubsection{Cell viability assay}

Cell viability was evaluated by MTT [3-(4,5-Dimethylthiazol-2-yl)-2,5-diphenyltetrazolium bromide ] assay $[19,20]$, which is based on the reduction of the yellow MTT by the mitochondrial dehydrogenase of intact viable or living cells to a purple formazan product. This reduction happens only when mitochondrial reductase enzymes are active. Therefore, increased purple colour is directly related to the number of viable cells. The amount of purple formazan produced by cells treated with compounds are compared with the amount of formazan produced by untreated control cells.

Cell viability was determined by MTT assay. Briefly, cells were grown in 96-well plates at a density of $3 \times 103$ cells per well and subjected to different concentrations of the compounds $(1000,500,250,125,62.5,31.2$, $15.67 .8,3.9$ and $1.9 \mu \mathrm{M})$. After $24 \mathrm{~h}$ incubation, MTT solution was added to reach a final concentration of 0.5 $\mathrm{mg} / \mathrm{mL}$. The cells were incubated for another $3 \mathrm{~h}$. Then current medium was removed and $100 \mu \mathrm{L}$ of DMSO solution was added. The absorbance was measured at $540 \mathrm{~nm}$ using a Cytation 3 Cell Imaging Multi-Mode Reader (Bio-Tek). Cell survival rates were expressed as the percentage of the DMSO (0.1\%) solvent control. 


\subsection{ADME properties}

All synthesized compounds were evaluated for their physicochemical properties using Swissadme online server (http://www.swiss adme.ch/) for calculations [21]. Furthermore, drug-like properties such as Lipinski rule of five of all compounds were tested also.

Acknowledgements: This research was partly presented at the 6th BBBB Conference on Pharmaceutical Sciences, 10-12 September, 2015, Helsinki, Finland.

Author contributions: Concept - F.T., B.K.; Design - F.T., B.K.; Supervision - F.T., B.K.; Resources - F.T., E.K.T., M.D., B.K.; Materials - F.T., E.K.T., M.D., B.K.; Data Collection and/or Processing - F.T., E.K.T., M.D., B.K.; Analysis and/or Interpretation - F.T., E.K.T., M.D., B.K.; Literature Search - F.T., B.K.; Writing - F.T., E.K.T., M.D., B.K.; Critical Reviews - F.T., E.K.T., M.D., B.K.

Conflict of interest statement: The authors declared no conflict of interest.

\section{REFERENCES}

[1] Ferlay J, Ervik M, Lam F, Colombet M, Mery L, Pineros M, Znaor A, Soerjomataram I, Bray F. Global Cancer Observatory: Cancer Today. Lyon, France: International Agency for Research on Cancer. https://gco.iarc.fr/today, (accessed June 18, 2021).

[2] Sung H, Ferlay J, Siegel R, Laversanne M, Soerjomataram I, Jemal A, Bray F. Global Cancer Statistics 2020: GLOBOCAN Estimates of Incidence and Mortality Worldwide for 36 Cancers in 185 Countries. CA Cancer J Clin. 2021; 71: 209-249. [CrossRef]

[3] Bray F, Ferlay J, Soerjomataram I, Siegel R, Torre LA, Jemal A. Global Cancer Statistics 2018: GLOBOCAN Estimates of Incidence and Mortality Worldwide for 36 Cancers in 185 Countries. CA Cancer J Clin. 2018; 68: 394-424. [CrossRef]

[4] Abdulnabi NM, Razzaq Al-Obaidy MMA, Tomi IH, Jaffer HJ. New calamitic mesogens derived from a furan ring: Synthesis, characterization and study of their mesomorphic behavior. J Mol Liq. 2021; 325: 114562. [CrossRef]

[5] Zhang H, Liu C. Synthesis and properties of furan/thiophene substituted difluoroboron b-diketonate derivatives bearing a triphenylamine moiety. Dyes Pigments. 2017; 143: 143-150. [CrossRef]

[6] Che J, Zheng C, Song MX, Bi Y, Liu Y, Li Y, Wu Y, Sun P, Piao H. Synthesis and antibacterial evaluation of furan derivatives bearing a rhodanine moiety. Med Chem Res. 2014; 23: 426-435. [CrossRef]

[7] Dige NC, Mahajan PG, Raza H, Hassan M, Vanjare BD, Hong H, Lee KH, Latip J, Seo S. Ultrasound mediated efficient synthesis of new 4-oxoquinazolin-3(4H)-yl)furan-2-carboxamides as potent tyrosinase inhibitors: Mechanistic approach through chemoinformatics and molecular docking studies. Bioorg Chem. 2019; 92: 103201. [CrossRef]

[8] Chohan S, Booysen IN, Mambanda A, Akerman MP. Formation, characterization and electrochemical properties of novel tetrasubstituted cobalt phthalocyanines bearing tetrahydropyran, furan and coumarin moieties. Inorg Chim Acta. 2016; 447: 183-191. [CrossRef]

[9] Li X, Yang X, Cui Z, Li Y, He H, Ling Y. Synthesis and Bioactivity of Novel N,N'-Diacylhydrazine Derivatives Containing Furan (II). Chin J Chem. 2010; 28: 1233-1239. [CrossRef]

[10] Sun G, Sun Z, Yang M, Liu X, Ma Y, Wei Y. Design, Synthesis, Biological Activities and 3D-QSAR of New N,N'Diacylhydrazines Containing 2,4-Dichlorophenoxy Moieties. Molecules. 2013; 18: 14876-14891. [CrossRef]

[11] Tapanyiğit O, Demirkol O, Güler E, Erşatır M, Çam ME, Giray ES. Synthesis and investigation of anti-inflammatory and anticonvulsant activities of novel coumarin-diacylated hydrazide derivatives. Arab J Chem. 2020; 13: 9105-9117. [CrossRef]

[12] Khan I, Tantray MA, Hamid H, Alam MS, Kalam A, Dhulap A. Synthesis of benzimidazole based thiadiazole and carbohydrazide conjugates as glycogen synthase kinase-3b inhibitors with antidepressant activity. Bioorg Med Chem Lett. 2016; 26: 4020-4024. [CrossRef]

[13] Cui Z, Huang J, Li Y, Ling Y, Yang X, Chen F. Synthesis and Bioactivity of Novel N,N'-Diacylhydrazine Derivatives Containing Furan(I). Chin . Chem. 2008; 26(5): 916-922. [CrossRef]

[14] Shafeeulla RM, Krishnamurthy G, Bhojynaik HS, Shivarudrappa HP, Shiralgi Y. Spectral thermal cytotoxic and molecular docking studies of $N^{\prime}$-2-hydroxybenzoyl; pyridine-4-carbohydrazide its complexes. Beni-Suef Univ J Basic Appl Sci. 2017; 6(4): 332-344. [CrossRef] 
[15] Sun J, Lv PC, Guo FJ, Wang XY, Han X, Zhang Y, Sheng GH, Qian SS, Zhu HL. Aromatic diacylhydrazine derivatives as a new class of polo-like kinase 1 (PLK1) inhibitors. Eur J Med Chem. 2014; 81: 420-426. [CrossRef]

[16] Xu F, Jio Y, Wen Q, Wang X, Zhang L, Yang K, Xu W. Synthesis and biological evaluation of N-(4-hydroxy-3mercaptonaphthalen-1-yl)amides as inhibitors of angiogenesis and tumor growth. Eur J Med Chem. 2013; 64: 377388. [CrossRef]

[17] Koçyiğit-Kaymakçığlu B, Yazıcı SS, Tok F, Dikmen M, Engür S, Oruç-Emre EE, İyidoğan A. Synthesis and anticancer activity of new hydrazide-hydrazones and their Pd (II) complexes. Lett Drug Des Discov. 2019; 16: 522-532. [CrossRef]

[18] Tok F, Koçyiğit-Kaymakçığlu B, İlhan R, Yılmaz S, Ballar-Kırmızıbayrak P, Taşkın-Tok T. Design, synthesis, biological evaluation and molecular docking of novel molecules to PARP-1 enzyme. Turk J Chem. 2019; 43: 12901305. [CrossRef]

[19] Mosmann T. Rapid colorimetric assay for cellular growth and survival: application to proliferation and cytotoxicity assays. J Immunol Methods. 1983; 65(1-2): 55-63. [CrossRef]

[20] Dikmen M, Özturk N, Özturk Y. The Antioxidant Potency of Punicagranatum L. Fruit Peel Reduces Cell Proliferation and Induces Apoptosis on Breast Cancer. J Med Food. 2011; 14(12): 1638-1646. [CrossRef]

[21] Daina A, Michielin O, Zoete V. SwissADME: A free web tool to evaluate pharmacokinetics, drug-likeness and medicinal chemistry friendliness of small molecules. Sci Rep. 2017; 7: 42717. [CrossRef]

This is an open access article which is publicly available on our journal's website under Institutional Repository at http://dspace.marmara.edu.tr. 